\title{
BMJ Open Longitudinal cohort survey of women's smoking behaviour and attitudes in pregnancy: study methods and baseline data
}

\author{
Sophie Orton, ${ }^{1,2,3}$ Katharine Bowker, ${ }^{1,2,3}$ Sue Cooper, ${ }^{1,2,3}$ Felix Naughton, ${ }^{2,4}$ \\ Michael Ussher, ${ }^{2,5}$ Kate E Pickett, ${ }^{2,6}$ Jo Leonardi-Bee, ${ }^{2,3,7}$ Stephen Sutton, ${ }^{2,4}$ \\ Nafeesa N Dhalwani, ${ }^{1,2,3,7}$ Tim Coleman $^{1,2,3}$
}

To cite: Orton S, Bowker K, Cooper S, et al. Longitudinal cohort survey of women's smoking behaviour and attitudes in pregnancy: study methods and baseline data. BMJ Open 2014;4:e004915. doi:10.1136/bmjopen-2014004915

- Prepublication history for this paper is available online. To view these files please visit the journal online (http://dx.doi.org/10.1136/ bmjopen-2014-004915).

Received 23 January 2014 Revised 7 April 2014 Accepted 28 April 2014

CrossMark

For numbered affiliations see end of article.

Correspondence to

Sophie Orton;

sophie.orton@nottingham.ac.uk

\section{ABSTRACT}

Objectives: To report the methods used to assemble a contemporary pregnancy cohort for investigating influences on smoking behaviour before, during and after pregnancy and to report characteristics of women recruited.

Design: Longitudinal cohort survey.

Setting: Two maternity hospitals, Nottingham, England.

Participants: 3265 women who attended antenatal ultrasound scan clinics were offered cohort enrolment; those who were 8-26 weeks pregnant and were currently smoking or had recently stopped smoking were eligible. Cohort enrollment took place between August 2011 and August 2012.

\section{Primary and secondary outcome measures:}

Prevalence of smoking at cohort entry and at two follow-up time points (34-36 weeks gestation and 3 months postnatally); response rate, participants' sociodemographic characteristics.

Results: 1101 (33.7\%, 95\% Cl $32.1 \%$ to $35.4 \%)$ women were eligible for inclusion in the cohort, and of these $850(77.2 \%, 95 \% \mathrm{Cl} 74.6 \%$ to $79.6 \%)$ were recruited. Within the cohort, $57.4 \%$ (N=488, $95 \% \mathrm{Cl}$ $54.1 \%$ to $60.7 \%$ ) reported to be current smokers. Current smokers were significantly younger than exsmokers $(p<0.05)$, more likely to have no formal qualifications and to not be in current paid employment compared to recent ex-smokers $(p<0.001)$.

Conclusions: This contemporary cohort, which seeks very detailed information on smoking in pregnancy and its determinants, includes women with comparable sociodemographic characteristics to those in other UK cross-sectional studies and cohorts. This suggests that future analyses using this cohort and aimed at understanding smoking behaviour in pregnancy may produce findings that are broadly generalisable.

\section{BACKGROUND}

Maternal smoking in pregnancy causes substantial harm to the infants and mothers,

\section{Strengths and limitations of this study}

This longitudinal cohort has collected the most detailed information on influences and determinants of smoking in pregnancy for almost 20 years.

- Future cohort analyses will investigate determinants of and describe women's smoking behaviour and attitudes and their receptivity to help with stopping smoking during pregnancy and postnatally.

- Although recruitment was in Nottingham, cohort women have a demographic profile which appears broadly representative of other UK women who smoke in pregnancy.

increasing risks of miscarriage, stillbirth, prematurity, low birth weight, perinatal morbidity and mortality, neonatal or sudden infant death. ${ }^{1}$ There is growing evidence of the impact of smoking in pregnancy on children; associations have been found with childhood behavioural problems, ${ }^{2}{ }^{3}$ and being overweight ${ }^{4}$ or obese in childhood. ${ }^{5}$ In addition, the costs to the National Health Service (NHS) of adverse maternal and infant health outcomes related to smoking are estimated to be between £31.6 million and £87.5 million/year. ${ }^{6}$ Reducing smoking in pregnancy is therefore an NHS priority, with the government aiming to reduce rates from $14 \%$ in $2009 / 2010$ to less than $11 \%$ by $2015 .^{7}$

Between 1984 and 2000, UK studies found that $30-35 \%$ of women smoked during pregnancy. ${ }^{8-11}$ More recently smoking in pregnancy appears to have decreased, ${ }^{12}$ but it remains a significant problem, particularly among younger and more deprived women; pregnant women aged under 20 are four times more likely to smoke than those aged 
over 35 years. ${ }^{12}$ Furthermore, mothers in routine and manual occupations (eg, people working in sales, services, technical, operative or agricultural jobs) are five times more likely to smoke during pregnancy than those in managerial and professional occupations. ${ }^{12}$ In 2001, the Millennium Cohort Survey (MCS) data showed that $35.3 \%$ of UK women smoked at some point during pregnancy, and $28.4 \%$ of women were smoking at 9 months postnatally. ${ }^{13}$ By 2010, the UK Infant Feeding Survey (IFS) showed that this had fallen to $26 \%$ of women smoking before or during pregnancy and $12 \%$ throughout $^{12}$; however, caution is required as IFS and MCS may not be completely comparable due to some differences in methodology and sampling. While both studies were UK-wide, the MCS collected retrospective maternal selfreport of smoking 9 months postnatally, disproportionately sampling families living in high poverty in Northern Ireland, Scotland and Wales, and from high ethnic minority populations in the UK. ${ }^{13}$ The IFS, however, collected maternal-reported smoking at 610 weeks postnatally from a representative sample of mothers weighted for age and deprivation. ${ }^{12}$

There is evidence that smoking behaviour fluctuates during pregnancy. In a US pregnancy cohort, up to $21 \%$ of quit attempts were made after the first trimester ${ }^{14}$ also, these were often repeated throughout pregnancy ${ }^{14}$ and $84 \%$ of pre-pregnancy smokers reported daily smoking later in pregnancy. ${ }^{15}$ However, little is known about smoking patterns in pregnancies in the UK because there have been very few cohort studies investigating this; in a 1986 cohort, $31 \%$ of pregnant mothers smoked and, although $25 \%$ of these were successful at quitting at some point during pregnancy, the timing of cessation was unclear and relapse to smoking was not reported. ${ }^{10} \mathrm{~A}$ second UK cohort conducted in the early $1990 \mathrm{~s}^{16}$ found complex smoking trajectories across pregnancy; just over $30 \%$ of smoking women stopped smoking temporarily in pregnancy, with quit attempts and relapse occurring at varying times across pregnancy.

Smoking therefore remains prevalent in pregnancy, and although smoking patterns appear to vary across pregnancy there is very little contemporary, normative data available. Similarly, almost nothing is known about when pregnant smokers are most receptive to offers of help with stopping smoking. Currently, pregnant women in the UK are systematically offered referral for NHS smoking cessation support during their first meeting with a midwife, but offers of support are less systematic in later pregnancy. Such later offers of support might be readily accepted but there is almost no research evidence on smokers' propensity for using cessation support during pregnancy. Research documenting women's smoking behaviour across pregnancy and how their attitudes to this and to receiving support with cessation might vary at different times in pregnancy could help determine when offers of cessation support made to pregnant women are most likely to be accepted. We have recruited a longitudinal, pregnancy cohort which has collected detailed information on these issues and also on the many potential determinants of and influences on smoking in pregnancy. The primary aim of this cohort study will be to estimate the proportion of smokers who initiate quit attempts in the second or third trimester of pregnancy. The secondary aims are to describe pregnant women's longitudinal smoking patterns throughout pregnancy, the timing of women's quit attempts and women's use of and attitudes to offers of NHS orientated cessation support and self-help cessation support. The longitudinal cohort will also explore whether individual, family and social context factors predict smoking patterns, use of and attitudes towards cessation support. We believe this cohort has collected some of the most detailed ever longitudinal UK data on smoking in pregnancy. Consequently, future analyses using cohort data will facilitate clearer understanding of the phenomenon of smoking in pregnancy.

We report methods used to assemble the cohort, details of the measures employed to quantify potential determinants of smoking, recruitment rates and the sociodemographic characteristics of cohort participants.

\section{METHODS}

\section{Participants}

Eligible women were those aged 16 years or above, and reported being between 8 and 26 weeks pregnant. Women who self-reported being either current smokers (defined as self-reported occasional smokers and daily smokers) or having smoked in the 3 months prior to becoming pregnant were eligible for participation. Women who were unable to understand study procedures sufficiently to provide consent (eg, due to cognitive difficulties) had previously enrolled in the study, or were unable to read or understand the written questionnaires in English were not enrolled.

\section{Recruitment and questionnaire distribution}

We recruited a longitudinal cohort of pregnant women using questionnaires completed at 8-26 weeks gestation, followed up at 34-36 weeks gestation, and 3 months after childbirth.

\section{Recruitment and baseline questionnaire}

On the basis of routine hospital data, there were approximately 10051 infants born in Nottingham hospitals in 2011/2012. We envisaged that at least 25\% of pregnant women in Nottingham would have smoked in the 3 months prior to or during pregnancy, providing 2500 potential participants from which we could recruit to the survey. Recruitment to the Pregnancy Lifestyle Survey took place between August 2011 and August 2012. Recruitment took place at two antenatal clinics within Nottingham University Hospitals NHS Trust (City Hospital and Queen's Medical Centre). Researchers attended on average five clinics per week; to ensure representative sampling researchers attended varied clinics 
and specialist clinics evenly distributed across both sites. All women self-reporting to be between 8 and 26 weeks gestation attending routine antenatal appointments at these clinics were invited to complete an anonymous screening questionnaire which determined study eligibility based on the criteria described above. Those who met the criteria were directed to read a participant information sheet describing the study, and, if willing, to then complete a baseline questionnaire; women could also seek further information from the researcher in the clinic.

On completion of the baseline questionnaire, women were offered a $£ 5$ high street shopping voucher as recognition for the time taken to complete the questionnaire. Written informed consent was obtained from those who wished to participate in the rest of the study and to complete the two further follow-up questionnaires. Researchers contacted any women who did not feel able to make a decision about participation while they were in the clinic after a further $24 \mathrm{~h}$ to ascertain whether they wished to take part.

\section{Follow-up at 34-36 weeks gestation}

Researchers liaised with hospital administration staff to routinely check antenatal hospital records ensuring that questionnaires were not sent to women who had died or whose fetuses/infants had died; for all other participants at this time point, a second questionnaire was sent by post, using the contact details provided at recruitment. In addition, participants who provided an email address were emailed a link to a web-based version of the questionnaire, and sent one email reminder. Web-based questionnaires were created using the Bristol Online Surveys tool. ${ }^{17}$ Participants were required to $\log$ in to the questionnaire using a unique ID number, details of which were provided in the email containing the URL link. The web-based questionnaires were designed with a similar layout to the paper versions and, with the exception of current smoking status, all questions were optional. Non-respondents were sent one postal/email reminder letter and then contacted by telephone; if no response was received, a text message reminder was sent to participants' mobile phones. Participants who were successfully contacted via telephone were invited to complete the questionnaire during the call.

All participants who completed follow-up questionnaires were sent a $£ 5$ shopping voucher.

\section{Follow-up at 3 months after childbirth}

Researchers liaised with hospital administration staff to routinely check antenatal hospital records to determine participants' actual delivery dates. A member of the research team sent the final questionnaire 3 months after the delivery date, using the same method as described above for follow-up in later pregnancy.

\section{Questionnaire contents}

Copies of the three questionnaires can be found in attached additional files, and a description of items selected from each is shown below. All questions used a range of response formats including yes/no responses, multiple choices and five-point Likert-type scales for attitudinal questions.

\section{Baseline questionnaire}

The baseline questionnaire contained 38 items including a combination of original questions and items derived from previous surveys or used in previous studies (shown by citations). The baseline questionnaire was divided into six sections: (1) screening questions, (2) your health and your pregnancy, (3) your smoking behaviour and beliefs, (4) your current smoking behaviour, (5) your interest in getting help to stop smoking and (6) about you (sociodemographic information). These questions asked women to describe their current smoking behaviour, ${ }^{18-21}$ nicotine dependence based on the 'heaviness of smoking index' ${ }^{20}$ general health, ${ }^{22-26}$ intentions to quit smoking and self-efficacy in achieving this, ${ }^{27} 28$ their beliefs about the harm smoking during pregnancy causes their baby, ${ }^{27}$ support from family and friends to stop smoking, ${ }^{27} 2930$ any stop smoking services accessed. ${ }^{27}$ The questionnaire also asked women about their opinions on a range of health professional provided and self-help stop smoking support, including telephone helplines, group sessions, one-to-one sessions, booklets, a DVD, websites, text messages, email support and a mobile phone/device application. ${ }^{31}$ The age that women left education, qualifications, whether they rented or owned their own home, access to a car or van within their household, employment status, occupation and ethnicity were also collected at baseline.

\section{Follow-up at 34-36 weeks gestation}

The first follow-up questionnaire contained 22 items, divided into four sections. Many of the questions from the baseline questionnaire were repeated, with the exclusion of screening and sociodemographic information already gathered at baseline. The four sections covered by follow-up questionnaire were (1) your smoking behaviour and beliefs, (2) your current smoking behaviour, (3) your interest in getting help to stop smoking and (4) your health and your pregnancy. In addition to the questions asked at baseline, this questionnaire also asked women about experiencing nausea or sickness during pregnancy ${ }^{32}$ and their concerns about weight gain as a result of stopping smoking. ${ }^{33}$

\section{Follow-up at 3 months after childbirth}

The second follow-up questionnaire contained 29 items, again divided into four sections. These were similar to the sections used in the baseline and first follow-up questionnaire, but the nature of the questions changed to reflect women's postnatal status. For example, the section (1) your smoking behaviour and beliefs asked women if they had smoked at all since the birth of their baby and focused on their confidence and 


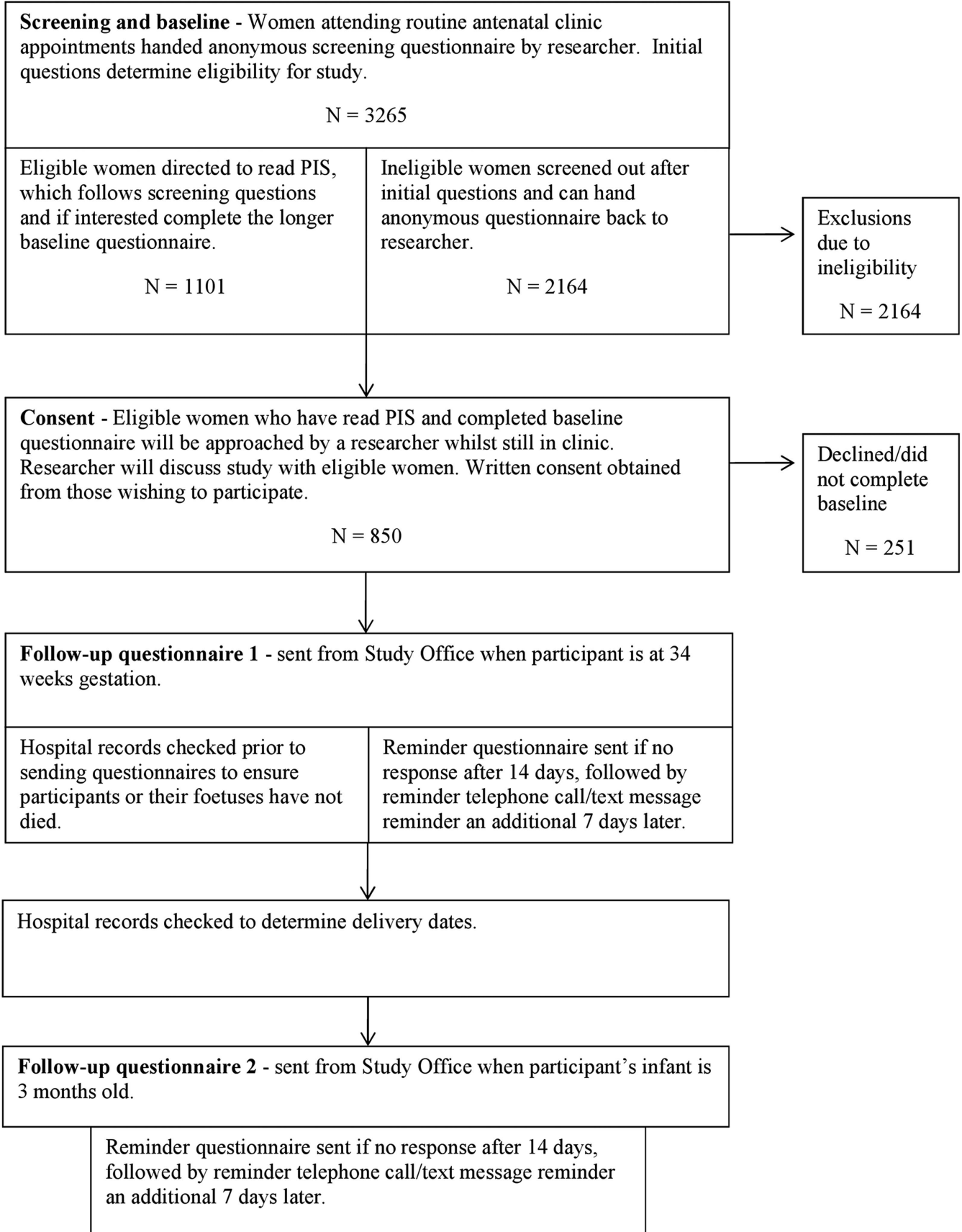

Figure 1 Flow diagram of recruitment and progress through study.

determination to stop smoking for good rather than until the birth of their baby. The final section (4) your health also asked women about smoking in the home and their beliefs about harm caused to infants through smoking in the home. In addition women were asked in this section about their relationship with their baby, ${ }^{26}$ confidence in their parenting ability, ${ }^{26}$ money concerns and family routine. ${ }^{34} 35$ All questions followed a similar format as the baseline and first follow-up questionnaire.

\section{Sample size}

The target sample size was 850 , anticipating a $20 \%$ drop out rate, giving an effective sample size of 683 pregnant smokers. The sample size calculation was conducted based on the primary aim of the cohort, to estimate the proportion of smokers who initiate quit attempts in the second or third trimester of pregnancy. This calculation estimated that 850 participants would be sufficient such that, if $20 \%$ of women reported quit attempts in the second or third trimester, we would be able to estimate this percentage with a $95 \%$ CI of $\pm 3 \%$.

\section{Data analysis}

Descriptive analyses were conducted summarising baseline cohort sociodemographic characteristics and information on current smoking behaviour from all women 
Table 1 Smoking status of all women who completed screening questionnaire

\begin{tabular}{|c|c|c|c|}
\hline & $N=3265$ & Per cent & $95 \% \mathrm{Cl}$ \\
\hline Never smoked & 1682 & 51.5 & 49.8 to 53.2 \\
\hline Completely stopped smoking more than 3 months before pregnancy & 460 & 14.1 & 12.9 to 15.3 \\
\hline Completely stopped smoking at some time in the 3 months prior to pregnancy & 86 & 2.6 & 2.1 to 3.2 \\
\hline Completely stopped smoking after finding out pregnant & 390 & 11.9 & 10.9 to 13.1 \\
\hline Smoke occasionally, not every day now pregnant & 153 & 4.7 & 4.0 to 5.4 \\
\hline Smoke everyday, cut down during pregnancy & 387 & 11.9 & 10.8 to 13.0 \\
\hline Smoke everyday, same as before pregnancy & 79 & 2.4 & 1.9 to 3.0 \\
\hline Smoke everyday, more than before pregnancy & 6 & 0.2 & 0.08 to 0.4 \\
\hline Missing & 22 & 0.7 & \\
\hline
\end{tabular}

approached, and those recruited into the cohort. $\chi^{2}$ Tests were used to examine potential differences in characteristics between those eligible women recruited and not recruited into the cohort, and current and recent ex-smokers within the cohort. Analyses were carried out in SPSS V.16.

Future analysis of the longitudinal cohort data will include descriptive statistics to delineate women's smoking patterns across pregnancy and receptivity to cessation support. Multivariable regression models will investigate whether patterns of smoking behaviour are predicted by individual, family or contextual factors and will be modelled for the potential impact of offering NHS Stop Smoking Services in Pregnancy (SSSP) and self-help to women at different points in pregnancy, based on the prevalence of women making unsupported quit attempts at those times.

\section{RESULTS}

\section{Sample characteristics}

Screening questionnaires were distributed and completed by 3265 women attending antenatal clinics in one of two sites at Nottingham University Hospitals NHS Trust. In total $148(4.5 \%)$ women approached declined to complete the screening questionnaire, giving us a response rate of $95.5 \%$ for screening questionnaires. Routine hospital data indicate that there were 10051 infants born in Nottingham hospitals in 2011/2012. We therefore estimate that just under one-third $(32.5 \%)$ of the pregnant population within Nottingham were screened. A flow diagram illustrating the recruitment and progression of participants through the study can be seen in figure 1 .

Table 1 shows the current smoking status of the 3265 women approached in antenatal clinics. In total $33.7 \%$ $(\mathrm{N}=1101,95 \%$ CI $32.1 \%$ to $35.4 \%)$ of women between 8 and 26 weeks gestation, and over 16 years of age, were either current smokers or recent ex-smokers (had stopped smoking either in the 3 months prior to becoming or after finding out they were pregnant). Overall $19.1 \%(\mathrm{~N}=625,95 \%$ CI $17.8 \%$ to $20.5 \%)$ of women who completed the screening questionnaire in clinic were currently smoking while pregnant.

\section{Cohort characteristics}

Of those eligible to participate, $87.7 \% \quad(\mathrm{~N}=966)$ completed the baseline survey, and $77.2 \% \quad(\mathrm{~N}=850)$ gave consent for participation in the longitudinal cohort survey. The cohort comprised of $26 \%$ of all women approached in antenatal clinics, and an estimated $8.5 \%$ of all pregnant women who gave birth within Nottingham in 2011/2012. A consort diagram detailing recruitment can be seen in figure 2 .

Those eligible women who completed the baseline questionnaire but did not consent to enter the longitudinal cohort $(\mathrm{N}=116,12.0 \%)$ were similar to the cohort in terms of smoking status, age, ethnicity, current employment and manual/non-manual occupations (table 2).

Table 3 shows the baseline smoking status of the 850 women enrolled into the cohort. In total $42.6 \%(\mathrm{~N}=362$, $95 \%$ CI $39.3 \%$ to $45.9 \%$ ) of participants reported having stopped smoking either in pregnancy or within the 3 months prior to this ('recent ex-smokers'), and $57.4 \%$ $(\mathrm{N}=488,95 \%$ CI $54.1 \%$ to $60.7 \%)$ reported to be current smokers.

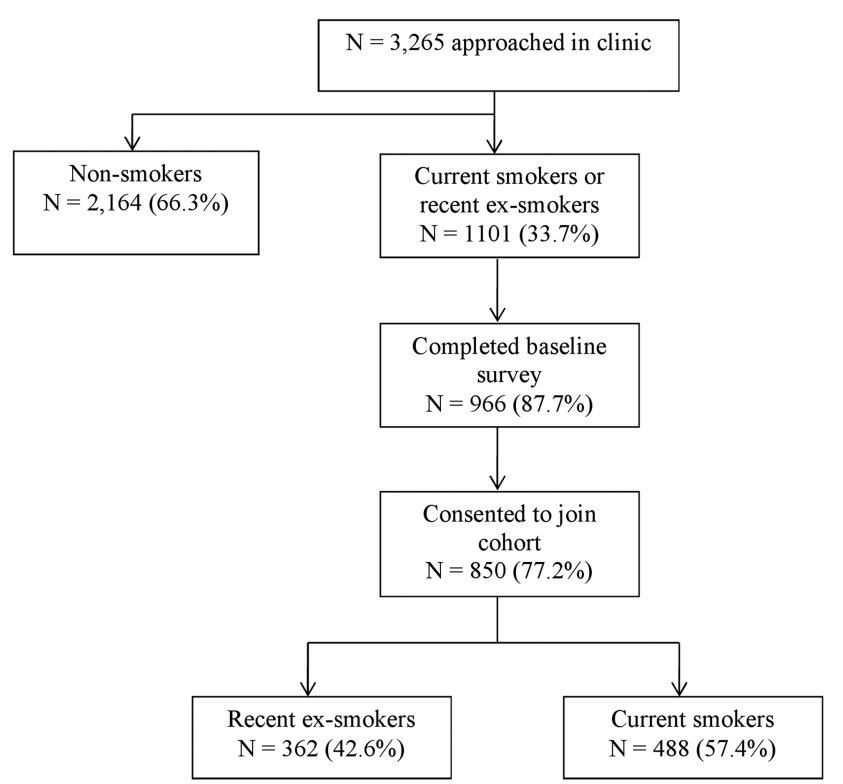

Figure 2 Consort diagram of recruitment. 
Table 2 Comparison of eligible women who consented and declined to enter cohort

\begin{tabular}{|c|c|c|c|c|c|}
\hline & \multicolumn{2}{|c|}{$\begin{array}{l}\text { Consented } \\
\mathrm{N}=850(88 \%)\end{array}$} & \multicolumn{2}{|c|}{$\begin{array}{l}\text { Declined } \\
\mathrm{N}=116(12 \%)\end{array}$} & \multirow[b]{2}{*}{ p Value } \\
\hline & $\mathbf{N}$ & Per cent & $\mathbf{N}$ & Per cent & \\
\hline \multicolumn{6}{|l|}{ Smoking status } \\
\hline Recent ex-smoker & 362 & 42.6 & 59 & 50.9 & \\
\hline Current smoker & 488 & 57.4 & 57 & 49.1 & 0.092 \\
\hline Mean age (SD) & 25.8 years & (SD 5.6) & 25.9 years & (SD 5.7) & \\
\hline \multicolumn{6}{|l|}{ Ethnicity } \\
\hline White British & 751 & 89.0 & 55 & 82.1 & \\
\hline Other ethnicity & 93 & 11.0 & 12 & 17.9 & 0.089 \\
\hline \multicolumn{6}{|l|}{ Home ownership } \\
\hline Own home & 166 & 19.6 & 15 & 23.1 & \\
\hline Do not own home & 680 & 80.4 & 50 & 76.9 & 0.501 \\
\hline \multicolumn{6}{|l|}{ Employment } \\
\hline In current paid work & 383 & 45.2 & 36 & 52.2 & \\
\hline Not in current paid work & 465 & 54.8 & 33 & 47.8 & 0.261 \\
\hline \multicolumn{6}{|c|}{ Current or most recent occupation manual/non-manual } \\
\hline Non-manual occupation & 216 & 28.2 & 22 & 38.6 & \\
\hline Manual occupation or not applicable & 549 & 71.8 & 35 & 61.4 & 0.096 \\
\hline
\end{tabular}

As seen in table 4, differences between current and recent ex-smokers were observed across a range of sociodemographic characteristics. Current smokers were significantly younger than ex-smokers $(p<0.05)$, more likely to have no formal qualifications, to have left full-time education at a younger age, to not own their homes, to not be in current paid employment and to not be in non-manual occupations compared with recent ex-smokers $(\mathrm{p}<0.001)$.

\section{DISCUSSION}

This is the first UK pregnancy cohort for 20 years to investigate smoking behaviour in pregnancy and, we believe, it may include more detailed longitudinal data on smoking and its determinants than any predecessor studies. We found that a third of women between 8 and 26 weeks gestation, and aged over 16 years, screened within Nottingham antenatal clinics were smoking either during pregnancy, or had smoked in the 3 months prior to this. Within our cohort of 850 pregnant women, we observed that $57 \%$ were current smokers and $43 \%$ had stopped either in pregnancy or 3 months prior to this. Current smokers entering our cohort were significantly younger, less educated and from lower socioeconomic backgrounds than recent ex-smokers. These findings are similar to those from previous UK cohorts, which reported that women who smoke before or during pregnancy are more likely to be less than 25 years of age, ${ }^{10} 12$ more likely to have left education at a younger age and gained fewer formal qualifications, ${ }^{10} 13 \quad 3637$ and to be from lower socioeconomic groups than non-smokers. ${ }^{10-13} 3637$

Twenty-four years ago, The Nottingham Mothers Stop Smoking Project surveyed women within Nottingham Hospitals, using similar definitions of smoking to those we used. ${ }^{10}$ Comparing current smoking rates to those recorded earlier, smoking rates appear to have declined substantially. Within this earlier cohort $64 \%$ of women smoked either before or during pregnancy and this was nearly the double rate in our 2012 sample $(31 \%) .{ }^{10}$ The reduction in smoking prevalence between Nottingham surveys is comparable to the fall in prevalence documented by the authoritative Infant Feeding Survey, suggesting that cohort findings are valid. ${ }^{12}$

Prevalence of smoking before or during pregnancy reported by the Infant Feeding Survey is lower than found in our cohort. However, while smoking rates in the East Midlands are, in general, low compared with other regions, ${ }^{38}$ rates in Nottingham city are relatively

\section{Table 3 Cohort baseline smoking status}

\begin{tabular}{lrrr}
\hline Smoking status at baseline & N=850 & Per cent & 95\% Cl \\
\hline Completely stopped smoking at some time in the 3 months prior to pregnancy & 61 & 7.2 & 3.6 to 9.1 \\
Completely stopped smoking after finding out pregnant & 301 & 32.2 to 38.6 \\
Smoke occasionally, not every day now pregnant & 117 & 13.8 & 11.6 to 16.2 \\
Smoke everyday, cut down during pregnancy & 304 & 35.8 & 32.6 to 39.0 \\
Smoke everyday, same as before pregnancy & 64 & 7.5 & 5.9 to 9.5 \\
Smoke everyday, more than before pregnancy & 3 & 0.4 & 0.01 to 1 \\
\hline
\end{tabular}


Table 4 Sociodemographic characteristics of smokers and recent ex-smokers in cohort

\begin{tabular}{|c|c|c|c|c|c|c|c|}
\hline \multirow[b]{2}{*}{ Demographic data } & \multicolumn{2}{|c|}{$\begin{array}{l}\text { Total } \\
\mathrm{N}=850\end{array}$} & \multicolumn{2}{|c|}{$\begin{array}{l}\text { Current } \\
\text { smokers } \\
\mathrm{N}=488\end{array}$} & \multicolumn{2}{|c|}{$\begin{array}{l}\text { Recent } \\
\text { ex-smokers } \\
\mathrm{N}=362\end{array}$} & \multirow{2}{*}{$\begin{array}{l}\text { Unadjusted } \\
\text { OR }(95 \% \mathrm{CI})\end{array}$} \\
\hline & $\mathbf{N}$ & Per cent & $\mathbf{N}$ & Per cent & $\mathbf{N}$ & Per cent & \\
\hline \multicolumn{8}{|l|}{ Age, years } \\
\hline$<20$ & 150 & 17.7 & 97 & 20 & 53 & 14.6 & $1.00^{*}$ \\
\hline $21-25$ & 309 & 36.5 & 179 & 36.9 & 130 & 35.9 & 0.75 (0.5 to 1.1$)$ \\
\hline $26-30$ & 215 & 25.4 & 123 & 25.4 & 92 & 25.4 & $0.73(0.48$ to 1.1$)$ \\
\hline $31-35$ & 118 & 13.9 & 62 & 12.8 & 56 & 15.5 & $0.7(0.37$ to 0.1$)$ \\
\hline $36-40$ & 51 & 6.0 & 22 & 4.5 & 29 & 8.0 & $0.42(0.22$ to 0.79$)$ \\
\hline Over 40 & 4 & 0.5 & 2 & 0.4 & 2 & 0.6 & 0.55 (0.8 to 3.99) \\
\hline \multicolumn{8}{|l|}{ Ethnicity } \\
\hline White British & 751 & 89 & 447 & 92 & 304 & 84.9 & $1.00^{*}$ \\
\hline White Irish/other white background & 32 & 3.8 & 14 & 2.9 & 18 & 5.0 & $0.53(0.26$ to 1.1$)$ \\
\hline Asian/Asian British & 9 & 1.1 & 2 & 0.4 & 7 & 2.0 & 0.19 (0.04 to 0.94$)$ \\
\hline Black/Black British & 7 & 0.8 & 1 & 0.2 & 6 & 1.7 & 0.11 (0.01 to 0.95$)$ \\
\hline Mixed background & 38 & 4.5 & 20 & 4.1 & 18 & 5.0 & 0.76 (0.39 to 1.45$)$ \\
\hline Other & 7 & 0.8 & 2 & 0.4 & 5 & 1.4 & $0.27(0.05$ to 1.4$)$ \\
\hline \multicolumn{8}{|l|}{ Qualifications held } \\
\hline No qualifications & 155 & 18.2 & 128 & 26.2 & 27 & 7.5 & $1.00^{\star *}$ \\
\hline $\begin{array}{l}\text { General Certificate of Secondary } \\
\text { Educations or equivalent }\end{array}$ & 355 & 41.7 & 213 & 43.7 & 142 & 39.2 & $0.32(0.2$ to 0.50$)$ \\
\hline AS/A-levels or equivalent & 174 & 20.5 & 81 & 16.6 & 93 & 25.7 & $0.18(0.11$ to 0.30$)$ \\
\hline Degree or equivalent & 133 & 15.6 & 42 & 8.6 & 91 & 25.1 & 0.1 (0.06 to 0.17$)$ \\
\hline Other & 33 & 2.9 & 24 & 4.9 & 9 & 2.5 & $0.56(0.24$ to 1.35$)$ \\
\hline \multicolumn{8}{|l|}{ Age left full time education, years } \\
\hline 16 and under & 469 & 56.4 & 307 & 64.9 & 162 & 45.25 & $1.00^{* *}$ \\
\hline $17-19$ & 219 & 26.4 & 112 & 23.68 & 107 & 29.89 & 0.55 (0.40 to 0.77$)$ \\
\hline 20 or older & 115 & 13.8 & 41 & 8.67 & 74 & 20.67 & 0.29 (0.19 to 0.45$)$ \\
\hline Still in full time education & 28 & 3.4 & 13 & 2.75 & 15 & 4.19 & 0.46 (0.21 to 0.99$)$ \\
\hline \multicolumn{8}{|l|}{ Home ownership } \\
\hline Own home & 166 & 19.6 & 57 & 11.8 & 109 & 30.1 & $1.00^{\star \star}$ \\
\hline Do not own home & 680 & 80.0 & 427 & 88.2 & 253 & 69.9 & 3.23 (2.26 to 4.6$)$ \\
\hline \multicolumn{8}{|l|}{ Current employment } \\
\hline In current paid work & 383 & 45.1 & 164 & 33.6 & 219 & 60.5 & $1.00^{* *}$ \\
\hline Not in current paid work & 467 & 54.9 & 324 & 66.4 & 143 & 39.5 & 3.03 (2.28 to 4.01$)$ \\
\hline \multicolumn{8}{|c|}{ Current or most recent occupation manual/non-manual } \\
\hline In non-manual occupation & 216 & 28.2 & 75 & 17.6 & 141 & 41.6 & $1.00^{* *}$ \\
\hline In manual occupation/not applicable & 549 & 71.8 & 351 & 82.4 & 198 & 58.4 & 3.33 (2.28 to 4.01$)$ \\
\hline
\end{tabular}

high. Smoking prevalence among Nottingham adults (non-pregnant) was reported as $27 \%$ in $2011^{39}$ and this is higher than the national average for England $(20 \%){ }^{38}$ Moreover, Nottingham City ranked 20 of 326 local authorities in England for deprivation in $2010 .^{40}$ Together, these factors are likely to contribute towards higher rates of smoking in pregnancy in Nottingham, again suggesting that cohort findings are valid.

Our cohort study found similar associations between smoking behaviour and demographic characteristics as reported in previous studies. For example, it has been widely reported that smoking in pregnancy is more prevalent in younger women. ${ }^{10} 12$ Previous cohorts have further shown smoking in pregnancy to be linked with lower socioeconomic status, whereby those pregnant women in routine or manual occupations are up to five times more likely to smoke. ${ }^{10-13}$ As with our cohort, Madeley et $a l^{10}$ and the $\mathrm{MCS}^{13}$ reported lower educational attainment to be strongly related to smoking in pregnancy. These studies observed high smoking rates in those who had left education at 16 years or younger, had lower than General Certificate of Secondary Education (GCSE)-level qualifications or no qualifications 10 37; similarly, we found that $60 \%$ of cohort women had no educational qualifications higher than GCSE, with current smokers having left full-time education at a younger age.

Comparisons between women who smoke in pregnancy and 'recent ex-smokers' gave similar findings in our sample and in the MCS. Smokers enrolled in the MCS were more likely to be in routine and semiroutine 
occupations, ${ }^{36}$ and less likely to be classified as 'nonworking class' compared with women who had stopped smoking early in pregnancy. ${ }^{37}$ Current smokers were also less likely to have achieved GCSE qualifications or above. ${ }^{37}$ Current smokers and those who had quit were similar in age. ${ }^{36}$ Findings from our cohort were very similar, with the exception that 'recent ex-smokers' were more likely to be older.

A characteristic of our cohort is that it predominantly consists of white British population. This is similar to previous cohorts, for example $87.1 \%$ of respondents within the MCS ${ }^{13}$ and $82 \%$ in the 2010 IFS were white British. ${ }^{41}$ Like our own cohort, the $\mathrm{MCS}^{13}$ found smoking during pregnancy to be more prevalent among women of white British ethnicity. With the exception of those of black Caribbean and Irish ethnicity (smoking prevalence of $24 \%$ and $26 \%$, respectively), smoking prevalence among women from ethnic minorities is generally low at less than $8 \% .{ }^{42}$ However, as the proportion of ethnic minorities within our cohort is low, the data, perhaps can be used most securely to form hypotheses about influences on smoking within a white British population.

A strength of our study was the very high response rate achieved, with $96 \%$ of women attending selected antenatal clinics within the Nottingham University Hospital Trust having their smoking status recorded and being screened for eligibility, accounting for around one-third of all births within Nottingham. Women who did not attend antenatal screening could not have been included in the cohort; however, 99\% of UK women attend ultrasound anomaly screening scans $^{43}$; so our methods are likely to provide a similar sample to that obtained from a thorough population-based approach. A further strength of our study was the prospective recording of smoking status during pregnancy; some previous cohorts collected data retrospectively during the postnatal period, ${ }^{12}{ }^{13}$ subjecting their findings to recall error and bias.

A potential limitation of this research and of our cohort was the reliance on self-reported smoking status data. The social stigma of smoking in pregnancy may lead to under-reporting and therefore a response bias but few studies have investigated this. ${ }^{44}$ In a Scottish study, self-reported smoking status measured at 8-12 weeks gestation was noted to be $25 \%$ lower than that measured by serum cotinine at 15-16 weeks gestation. ${ }^{45}$ This could have been due to under-reporting of smoking habits; however, it is also likely that at least a proportion of this was due to relapse to smoking as gestation progresses. However, other research has shown a high correlation between self-reported smoking and biomedical markers within pregnant populations, ${ }^{15}{ }^{46}$ suggesting that self-report measures can be a valid method of assessing smoking status in surveys such as ours. Furthermore, although recruitment was limited to Nottingham the observed demographic profile of smokers within the cohort is, given the composition of other cohorts, as expected and broadly representative of pregnant smokers generally.

This cohort provides contemporary data source for investigating the phenomenon of smoking in pregnancy. We achieved a high response rate which has resulted in comprehensive population coverage. Future analyses using cohort data will attempt to gain greater understanding of smoking in pregnancy and, as the characteristics of cohort participants are similar to those of other white British smokers, findings from future studies will be most generalisable pregnant smokers from this social group.

\section{Author affiliations}

${ }^{1}$ Division of Primary Care, University of Nottingham, Nottingham, UK

${ }^{2}$ UK Centre for Tobacco and Alcohol Studies, UK

${ }^{3}$ National Institute for Health Research, School for Primary Care Research, UK

${ }^{4}$ Behavioural Science Group, University of Cambridge, Cambridge, UK

${ }^{5}$ Division of Population Health Sciences and Education, St. Georges,

University of London, London, UK

${ }^{6}$ Department of Health Sciences, University of York, York, UK

${ }^{7}$ Epidemiology and Public Health, University of Nottingham, Nottingham, UK

Acknowledgements The authors would like to thank Nottingham University Hospital NHS Trust for facilitating this research. We also thank Rachel Whitemore for invaluable assistance in setting up this research. Tim Coleman acknowledges the support of the East Midlands Collaboration for Leadership in Applied Health Research and Care (CLARHC).

Contributors SO helped design the data collection process, recruited participants into the cohort and drafted and revised this manuscript. KB helped design the data collection process, recruited participants into the cohort and made substantial contributions to the preparation of this manuscript. SC helped conceive the study, made a substantial contribution to the development of the study protocol and questionnaires, assisted with day-to-day troubleshooting during the data-collection phase and contributed to the preparation of this manuscript. FN, MU, KEP, JL-B and SS all contributed to the development of the study protocol and questionnaires, contributing expertise in their own particular knowledge base, and to the preparation of this manuscript. NND contributed to the drafting and preparation of this manuscript. TC conceived the study and made substantial contributions to the development of the study protocol and questionnaires, and the preparation of this manuscript. All the authors read and approved the final manuscript.

Funding This article presents independent research funded by the National Institute for Health Research (NIHR) under the Programme Grants for Applied Research (RP-PG-0109-10020).

\section{Competing interests None.}

Ethics approval The study was approved by Derbyshire Research Ethics Proportionate Review Sub-Committee (reference number 11/EM/0078).

Provenance and peer review Not commissioned; externally peer reviewed.

Data sharing statement No additional data are available.

Open Access This is an Open Access article distributed in accordance with the Creative Commons Attribution Non Commercial (CC BY-NC 3.0) license, which permits others to distribute, remix, adapt, build upon this work noncommercially, and license their derivative works on different terms, provided the original work is properly cited and the use is non-commercial. See: http:// creativecommons.org/licenses/by-nc/3.0/

\section{REFERENCES}

1. Royal College of Physicians. Smoking and the young. A report of a working party of the Royal College of Physicians. London, 1992. 
2. Thapar A, Fowler T, Rice F, et al. Maternal smoking during pregnancy and attention deficit hyperactivity disorder symptoms in offspring. Am J Psychiatry 2003;160:1985-9.

3. Langley K, Rice F, van den Bree MBM, et al. Maternal smoking during pregnancy as an environmental risk factor for attention deficit hyperactivity disorder behaviour. A review. Minerva Pediatr 2005;57:359-71.

4. Oken E, Levitan EB, Gillman MW. Maternal smoking during pregnancy and child overweight: systematic review and meta-analysis. Int J Obes 2008;32:201-10.

5. Weng SF, Redsell SA, Swift JA, et al. Systematic review and meta-analyses of risk factors for childhood overweight identifiable during infancy. Arch Dis Child 2012;97:1019-26.

6. Godfrey $\mathrm{C}$, Pickett KE, Parrott S, et al. Estimating the costs to the NHS of smoking in pregnancy for pregnant women and infants. University of York, Public Health Research Consortium, 2010.

7. Department of Health. Healthy lives, healthy people: a tobacco control plan for England. London, 2011.

8. Martin J, White A, Britain G. Infant feeding, 1985: a survey carried out by social survey division of OPCS on behalf of the Department of Health and Social Security and the Scottish Home and Health Department. HM Stationery Office, 1988.

9. Rubin PC, Craig GF, Gavin K, et al. Prospective survey of use of therapeutic drugs, alcohol, and cigarettes during pregnancy. $\mathrm{Br} \mathrm{Med}$ $J$ (Clin Res Ed) 1986;292:81-3.

10. Madeley RJ, Gillies PA, Power FL, et al. Nottingham mothers stop smoking project-baseline survey of smoking in pregnancy. $J$ Public Health 1989;11:124-30.

11. Owen LA, Penn GL. Smoking and pregnancy: a survey of knowledge attitudes and behaviour 1992-1999. Health Education Authority, 1999.

12. The NHS Information Centre. Infant feeding survey 2010. Early results. The National Health Service Information Centre for Health and Social Care, 2011.

13. Dex S, Joshi H. Millennium Cohort Study First Survey: a user's guide to initial findings. Centre for Longitudinal Studies, Institute of Education, University of London, 2004.

14. Pickett KE, Wakschlag LS, Dai L, et al. Fluctuations of maternal smoking during pregnancy. Obstet Gynecol 2003;101:140-7.

15. Pickett KE, Rathouz PJ, Kasza K, et al. Self-reported smoking, cotinine levels, and patterns of smoking in pregnancy. Paediatr Perinat Epidemiol 2005;19:368-76.

16. Munafò MR, Heron J, Araya R. Smoking patterns during pregnancy and postnatal period and depressive symptoms. Nicotine Tob Res 2008;10:1609-20.

17. Bristol Online Surveys. Bristol Online Surveys. Secondary Bristol Online Surveys. http://www.survey.bris.ac.uk

18. Mullen PD, Carbonari JP, Tabak ER, et al. Improving disclosure of smoking by pregnant women. Am J Obstet Gynecol 1991;165:409-13.

19. Coleman T, Thornton J, Britton J, et al. Protocol for the smoking, nicotine and pregnancy (SNAP) trial: double-blind, placebo-randomised, controlled trial of nicotine replacement therapy in pregnancy 1186. BMC Health Serv Res 2007;7:2.

20. Heatherton TF, Kozlowski LT, Frecker RC, et al. Measuring the heaviness of smoking: using self-reported time to the first cigarette of the day and number of cigarettes smoked per day. $\mathrm{Br} J$ Addict 1989;84:791-9.

21. Sutton S, Gilbert H, Sutton S, et al. Effectiveness of individually tailored smoking cessation advice letters as an adjunct to telephone counselling and generic self-help materials: randomized controlled trial. Addiction 2007;102:994-1000.

22. UK Census. 2001 Census Questionnaires, England Individual Form 2001. http://www.ons.gov.uk/ons/guide-method/census/ census-2001/about-census-2001/census-2001-forms/index.html. Secondary 2001 Census Questionnaires, England Individual Form http://www.ons.gov.uk/ons/guide-method/census/census-2001/ about-census-2001/census-2001-forms/index.html

23. NICE. NICE, Antenatal and postnatal mental health; Clinical management and service guidance. Secondary NICE, antenatal and postnatal mental health; clinical management and service guidance. 2007. http://www.nice.org.uk/nicemedia/live/11004/30433/ 30433.pdf

24. Cohen S, Kamarck T, Mermelstein R. A global measure of perceived stress. J HealthSoc Behav 1983;24:385-96.
25. Cohen S, Williamson GM. Perceived stress in a probability sample of the United States. Soc Psychol Health 1988;1:31-67.

26. National Centre for Longitudinal Studies. Millennium Cohort Study, First Survey: CAPI Questionnaire Documentation. London, 2003.

27. Naughton F, Prevost AT, Gilbert H, et al. Randomized controlled trial evaluation of a tailored leaflet and SMS text message self-help intervention for pregnant smokers (MiQuit). Nicotine Tob Res 2012;14:569-77.

28. Dijkstra A, De Vries H. Do self-help interventions in health education lead to cognitive changes, and do cognitive changes lead to behavioural change? Br J Health Psychol 2001;6:121-34.

29. Bledsoe LK. Smoking cessation: an application of theory of planned behavior to understanding progress through stages of change. Addict Behav 2006;31:1271-6.

30. Rise J, Kovac V, Kraft P, et al. Predicting the intention to quit smoking and quitting behaviour: extending the theory of planned behaviour. Br J Health Psychol 2008;13:291-310.

31. Ussher M, West R, Hibbs N. A survey of pregnant smokers' interest in different types of smoking cessation support. Patient Educ Couns 2004:54:67-72.

32. Crystal SR, Bowen DJ, Bernstein IL. Morning sickness and salt intake, food cravings, and food aversions. Physiol Behav 1999;67:181-87.

33. Berg CJ, Park ER, Chang Y, et al. Is concern about post-cessation weight gain a barrier to smoking cessation among pregnant women? Nicotine Tob Res 2008;10:1159-63.

34. Booth CL, Mitchell SK, Barnard KE, et al. Development of maternal social skills in multiproblem families-effects on the mother child relationship. Dev Psychol 1989;25:403-12.

35. Barnard K, Hilsinger G, Patteson D, et al. Parent Protective Factors Project (1995-1999), Final Report. Seattle, WA: University of Washington, 1999.

36. Pickett KE, Wood C, Adamson J, et al. Meaningful differences in maternal smoking behaviour during pregnancy: implications for infant behavioural vulnerability. J Epidemiol Community Health 2008;62:318-24.

37. Pickett KE, Wilkinson RG, Wakschlag LS. The psychosocial context of pregnancy smoking and quitting in the Millennium Cohort Study. $J$ Epidemiol Community Health 2009;63:474-80.

38. The Health and Social Care Information Centre. Statistics on smoking: England 2012. The Health and Social Care Information Centre, 2012.

39. Nottingham City Council, NHS Nottingham City. Nottingham Citizens Survey 2011. Secondary Nottingham Citizens Survey 20112012. http://www.nottinghaminsight.org.uk/insight/library/citizens-survey. aspx

40. Department for Communities and Local Government. The English Indices of Deprivation 2010: Overall. Secondary The English Indices of Deprivation 2010: Overall 2011. https://www.gov.uk/government/ publications/english-indices-of-deprivation-2010

41. Health and Social Care Information Centre. Infant Feeding Survey 2010, 2012. Available from http://www.hscic.gov.uk/catalogue/ PUB08694/Infant-Feeding-Survey-2010-Consolidated-Report.pdf (accessed 9 May 2014).

42. Millward $D$, Karlson $S$. Tobacco use among minority ethnic populations and cessation interventions. A Race Equality Foundation Briefing Paper. Secondary Tobacco use among minority ethnic populations and cessation interventions. A Race Equality Foundation Briefing Paper 2011. http://www.better-health.org.uk/sites/default/ files/briefings/downloads/health-brief22 0.pdf

43. National perinatal epidemiology unit. Delivered with care: a national survey of women's experience of maternity care 2010. 2010. file:/// C:/Users/mczso/Downloads/Maternity-Survey-Report-2010.pdf (accessed 9 May 2014).

44. Shipton D, Tappin DM, Vadiveloo T, et al. Reliance on self-reported smoking during pregnancy underestimates smoking prevalence and reduces the reach of specialist cessation services: results from a retrospective, cross-sectional study. BMJ 2009;339:4347-54.

45. Shipton D, Tappin DM, Vadiveloo T, et al. Reliability of self reported smoking status by pregnant women for estimating smoking prevalence: a retrospective, cross sectional study. BMJ 2009;339: b4347.

46. George L, Granath F, Johansson ALV, et al. Self-reported nicotine exposure and plasma levels of cotinine in early and late pregnancy. Acta Obstet Gynecol Scand 2006;85:1331-7. 


\section{Correction}

Orton S, Bowker K, Cooper S, et al. Longitudinal cohort survey of women's smoking behaviour and attitudes in pregnancy: study methods and baseline data. BMJ Open 2014;4:e004915.

The final sentence was omitted from the Funding section. The full funding section should read:

"Funding This work was funded by the National Institute for Health Research (NIHR) under its Programme Grant for Health Research scheme (grant reference RP-PG-0109-10020). The views expressed are those of the authors and not necessarily those of the NHS, the NIHR or the Department Health."

BMJ Open 2015;5:e004915corr1. doi:10.1136/bmjopen-2014-004915corr1

CrossMark 